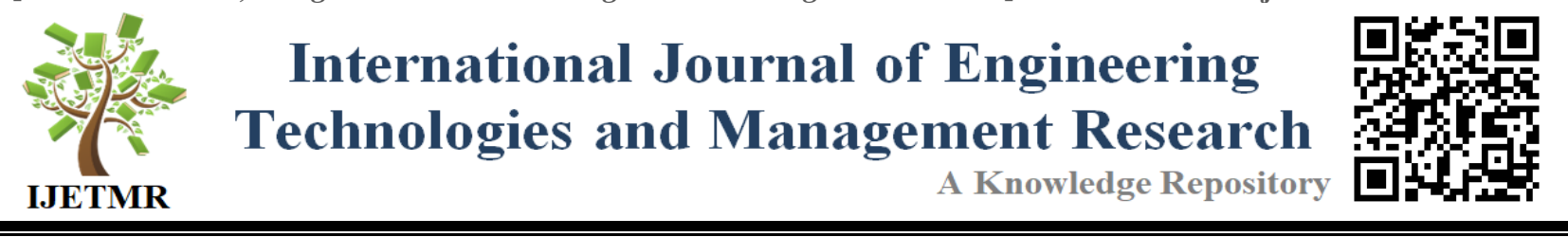

\title{
HIGH SPEED ADDER USING GDI TECHNIQUE
}

Merrin Mary Solomon ${ }^{1}$, Neeraj Gupta ${ }^{2}$, Rashmi Gupta ${ }^{2}$

${ }^{1}$ M.Tech Student, Amity School of Engineering \& Technology Amity University Haryana, Gurugram, India

${ }^{2}$ Assistant Professor, Amity School of Engineering \& Technology Amity University Haryana, Gurugram, India

Abstract:

Full adder is an important component for designing a processor. As the complexity of the circuit increases, the speed of operation becomes a major concern. Nowadays there are various architectures that exist for full adders. In this paper we will discuss about designing a low power and high speed full adder using Gate Diffusion Input technique. GDI is one of the present day methods through which one can design logical circuits. This technique will reduce power consumption, propagation delay, and area of digital circuits as well as maintain low complexity of logic design. The performance of the proposed design is compared with the contemporary full adder designs.

Keywords: Gate Diffusion Input; Full Adder; Power Dissipation; CMOS Logic; Delay.

Cite This Article: Merrin Mary Solomon, Neeraj Gupta, and Rashmi Gupta. (2018). "HIGH SPEED ADDER USING GDI TECHNIQUE." International Journal of Engineering Technologies and Management Research, 5(2:SE), 130-136. 10.29121/ijetmr.v5.i2.2018.634.

\section{Introduction}

In many of the VLSI applications such as Digital Image Processing (DSP), microprocessors and many more, we see the substantial use of adders. The arithmetic operations such as addition, subtraction, division, multiplication are all performed with the help of adders. Hence adders play an important role in the performance of the system and so designers are vigorously working in improving the performance of adders to enhance the overall performance. The use of portable devices such as laptops, mobile phones are increasing on an exponential rate. The power available for these portable devices is limited. Researchers have been extensively working on designing low power systems as it has been a major design parameter now days. Researchers have come up with different full adder designs that can bring about systems with high performance and low power utilization.

Here we are going to discuss few of the existing full adder designs like the conventional CMOS full adder, CPL full adder, ULP full adder, hybrid full adder and circuit.We will compare the performance of these full adders with the performance of the proposed full adder circuit. Comparison will be on the parameters of power, delay and the product of power and delay. Section II the full adder designs, section III the proposed design using GDI technique and the section IV the simulation result and comparison and finally conclusion. 
Full adder is a combinational circuit which has 3 inputs bits .It performs addition operation. Basically it has 3 inputs and 2 outputs. The Fig 1 shows the logical structure of full adder. Here we can see the 3 inputs are A, B and Cin and the outputs are expressed using $\operatorname{Sum}(\mathrm{S})$ and carry (Cout).Table 1 gives the truth table of a 1-bit full adder. The Boolean equation of the full adder is given by,

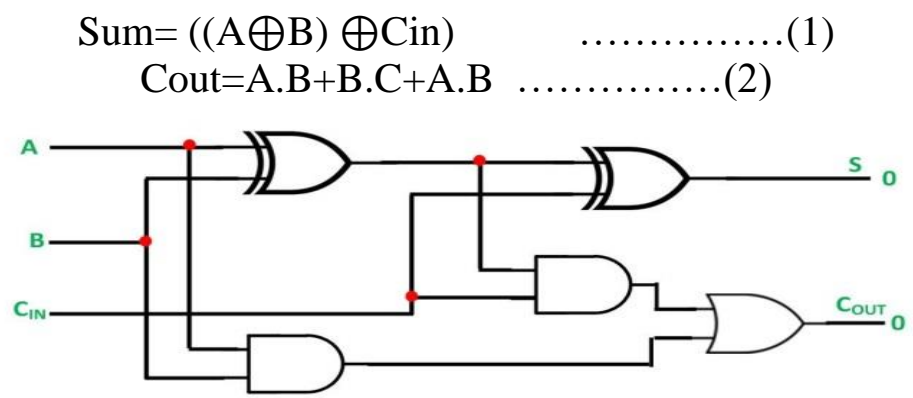

Figure 1: Full adder circuit

A full adder can be designed using two half adders. A full adder can be used to construct different counters such as ripple carry counter to add n-bit number. Full adders are generally connected in a cascade manner to build various logical circuits. [4]

Table 1: Truth Table of Full Adder

\begin{tabular}{|l|l|l|l|l|}
\hline A & B & Cin & Sum & Cout \\
\hline 0 & 0 & 0 & 0 & 0 \\
\hline 0 & 0 & 1 & 1 & 0 \\
\hline 0 & 1 & 0 & 1 & 0 \\
\hline 0 & 1 & 1 & 0 & 1 \\
\hline 1 & 0 & 0 & 1 & 0 \\
\hline 1 & 0 & 1 & 0 & 1 \\
\hline 1 & 1 & 0 & 0 & 1 \\
\hline 1 & 1 & 1 & 1 & 1 \\
\hline
\end{tabular}

\section{Existing Full Adder Topologies}

\subsection{Conventional CMOS Full Adder}

The conventional CMOS full adder consists of 28 transistors and is based on conventional CMOS structure. The structure consist of PMOS pull-up and NMOS pull-down transistors. 

Fig 2 shows the structure of CMOS full adder. The drawbacks of this circuit are that it produces unwanted delays because sum realizes on Cout signal, it dissipates more power and since the number of transistors is high, it occupies larger area.[2]

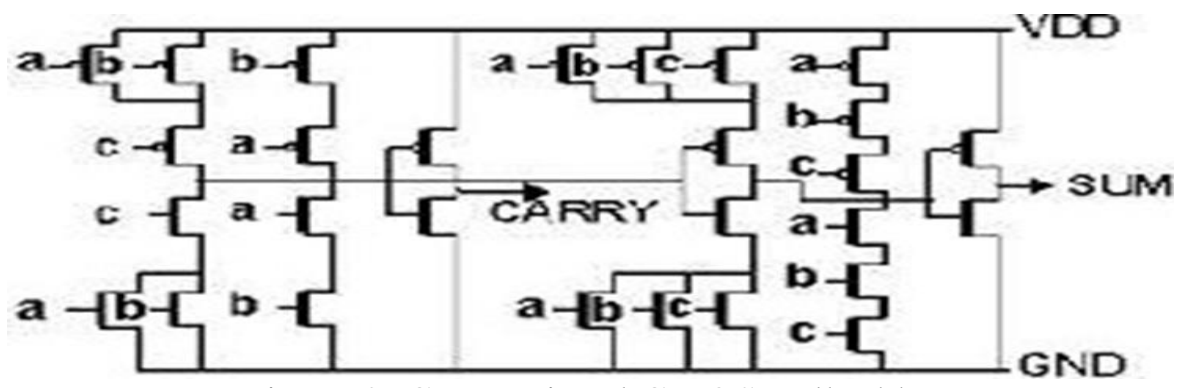

Figure 2: Conventional CMOS Full adder

\subsection{CPL Full Adder}

Complementary Pass transistor logic (CPL) based full adder circuit. It comprises of complimentary inputs/outputs, a NMOS pass transistor circuit and CMOS output inverters. In addition to this, PMOS keeper circuit is used to reduce static power consumption and also for swing restoration. The system basically consists of two stage pass transistor network. In the first stage, the network is designed by applying true inputs to pass transistor logic and second stage is designed by applying complementary inputs to pass transistor logic. The drawback of CPL full adder is that the number of transistors used is more. Here 32 transistors are used and hence it occupies more area. Another drawback is that in the NMOS network swing-reduction has to be restored. Fig 3 shows the structure of CPL full adder.
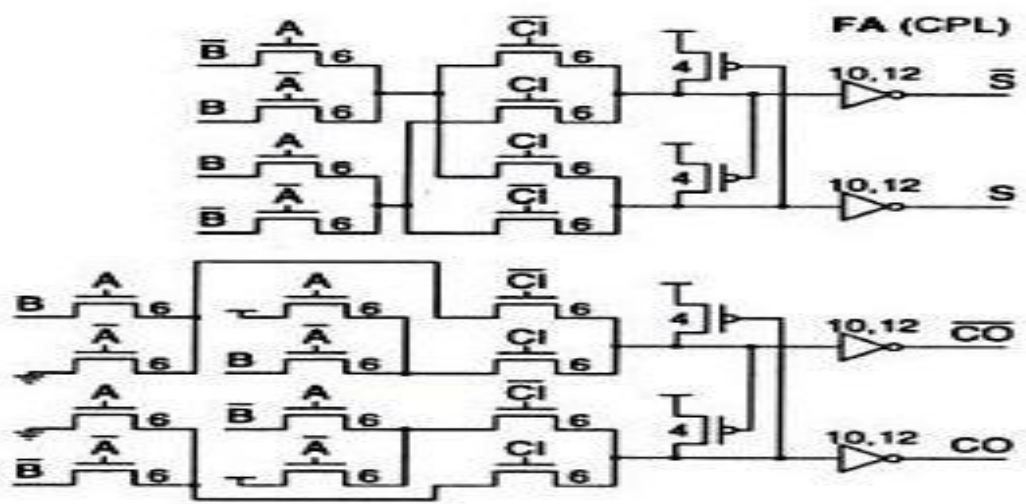

Figure 3: CPL full adder

\subsection{Hybrid Full Adder}

As the name suggest it is a combination of multiple logics. It uses completely different logics to improve the performance of the full adder. Hybrid full adder uses a LP XOR/XNOR gates and output inverters. To prevent the voltage step that takes place in 0 to 1 transitions in the sum output signal and also to acquire reduced dynamic power consumption additional optimization has been done. Fig 4 shows the design of hybrid full adder. The number of transistors used here is less compared to the other complementary full adders. Only 20 transistors are used. The speed 
of this circuit is comparatively high when comparing to the other counterparts and this is because the critical path consists of only two transistors.

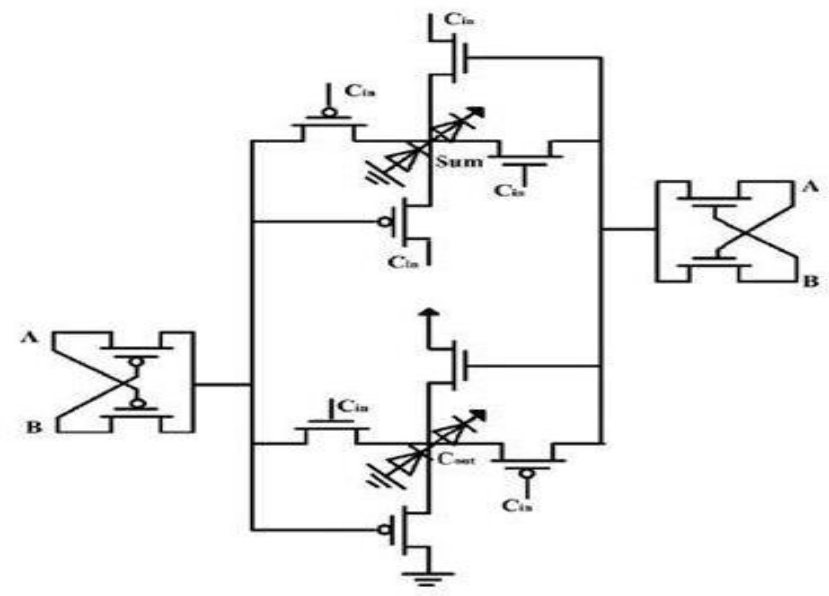

Figure 4: Hybrid Full adder

\subsection{ULP Full Adder}

The ULP full adder uses CMOS logic as well as pass-transistor logic for designing the full adder. The main feature of this circuit is the addition of a unique voltage restorer, ULP diode. It will eliminate the need for speed compensation at the output. Fig 5 shows the design of ULP full adder. This circuit design is resilient against voltage scaling and also it is difficult to implement transistor sizing. Since the design comprises of two different logics, it is non-symmetrical and irregular in design. [1], [3]

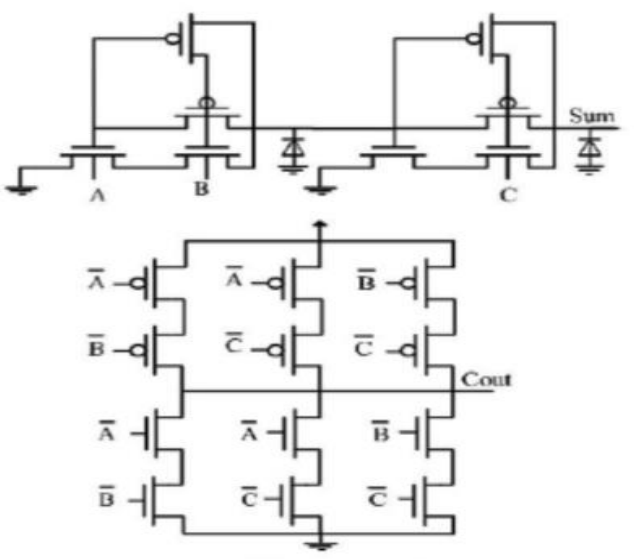

Figure 5: ULP Full adder

\section{Proposed Design}

The proposed system designs full adder circuit using Gate diffusion Input (GDI) technique. Using this technique one can design a digital circuit with low power in embedded system. The number of transistors used in the circuit is minimum hence they are used by the circuit is reduced as well as the delay and power consumption. [7] Compared to the existing conventional designs 
GDI technique is much efficient. Even though the technique has many advantages, there are few drawbacks which can be rectified. A GDI cell consists of 3 inputs. One input G which is the gate input for PMOS and NMOS, $\mathrm{P}$ which is the input to source/drain of PMOS and $\mathrm{N}$ which is the input to source/drain of NMOS.

The major benefit of using GDI technique is that a large number of functions can be implemented using this technique. We can see from the table 2 that GDI can be used for implementing various designs such as MUX, AND, OR etc. The most complex design among these is the designing of MUX, which can be done using 2 transistors. Whereas using other conventional techniques it requires 8-10 transistors for designing a MUX. The main drawback of GDI technique is that of swing degradation. This is due to threshold loss and to eliminate this we have to use silicon on insulator or twin-well process to realize, which is very expensive. Designing a full adder the major building block is XOR gate using GDI technique. The design is shown in the Fig 7.

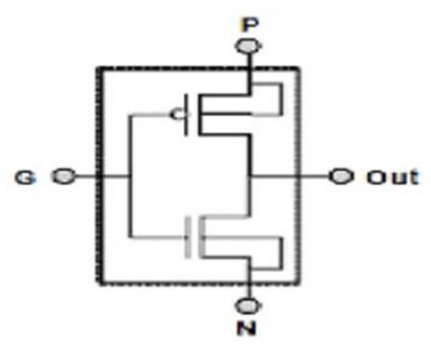

Figure 6: GDI cell

The major benefit of using GDI technique is that a large number of functions can be implemented using this technique. We can see from the table 2 that GDI can be used for implementing various designs such as MUX, AND, OR etc. The most complex design among these is the designing of MUX, which can be done using 2 transistors. Whereas using other conventional techniques it requires 8-10 transistors for designing a MUX. The main drawback of GDI technique is that of swing degradation. This is due to threshold loss and to eliminate this we have to use silicon on insulator or twin-well process to realize, which is very expensive. Designing a full adder the major building block is XOR gate using GDI technique. The design is shown in the Fig 7.

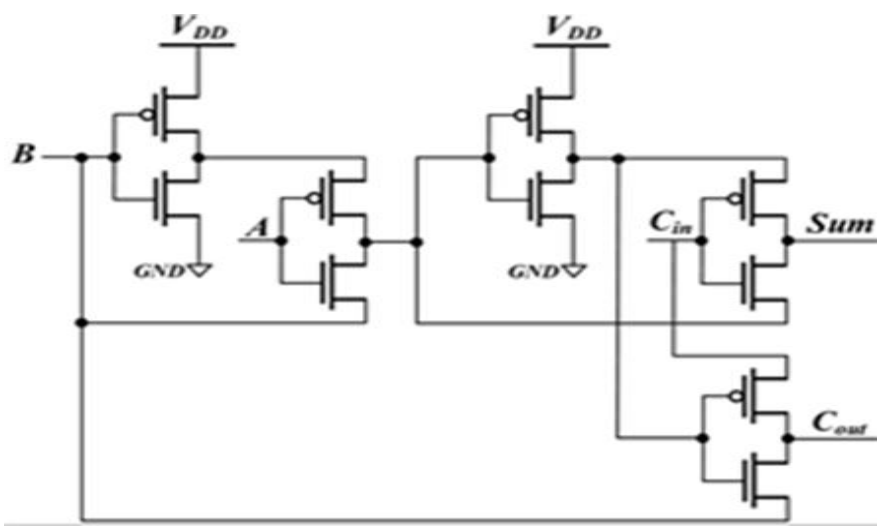

Figure 7: Full adder using GDI 


\begin{tabular}{|c|c|c|c|l|}
\hline $\mathbf{N}$ & $\mathbf{P}$ & $\mathbf{G}$ & OUT & FUNCTION \\
\hline 0 & $\mathrm{~B}$ & $\mathrm{~A}$ & $\bar{A} \mathrm{~B}$ & $\mathrm{~F} 1$ \\
\hline $\mathrm{B}$ & 1 & $\mathrm{~A}$ & $\bar{A}+\mathrm{B}$ & $\mathrm{F} 2$ \\
\hline 1 & $\mathrm{~B}$ & $\mathrm{~A}$ & $\mathrm{~A}+\mathrm{B}$ & $\mathrm{OR}$ \\
\hline $\mathrm{B}$ & 0 & $\mathrm{~A}$ & $\mathrm{AB}$ & $\mathrm{AND}$ \\
\hline $\mathrm{C}$ & $\mathrm{B}$ & $\mathrm{A}$ & $\bar{A} \mathrm{~B}+\mathrm{AC}$ & $\mathrm{MUX}$ \\
\hline 0 & 1 & $\mathrm{~A}$ & $\bar{A}$ & $\mathrm{NOT}$ \\
\hline
\end{tabular}

In here 5 PMOS and 5 NMOS transistors are used. In total only 10 transistors are used to design the full adder. A, B, Cin are the input and Cout and sum are the output produced.

\section{Comparison and Analysis}

Table 3: Performance Analyses

\begin{tabular}{|l|l|l|l|l|}
\hline & No. Of Transistors & Delay(ps) & Power( $\boldsymbol{\mu w})$ & PDP \\
\hline CMOS & 28 & 98.46 & 3.903 & 0.384 \\
\hline CPL & 32 & 110 & 3.91 & 0.0431 \\
\hline ULP & 20 & 22.66 & 2.73 & 0.061 \\
\hline Hybrid & 20 & 6.706 & 4.103 & 0.027 \\
\hline GDI & 10 & 31.48 & 2.449 & 0.077 \\
\hline
\end{tabular}

Here we have discussed about 5 full adders including the proposed system. We are comparing these adders on the basis of three criterions power, delay and the number of transistors used. The resultant power and delay value can be used to find the power delay product (PDP). From the table 3 , it is clear that the better full adder is the proposed full adder which is designed using GDI technique. The number of transistors used in GDI technique is minimum compared to other full adders used here. The power dissipation of full adder designed using GDI technique is the least when compared to the other discussed full adders.

\section{Conclusion}

The goal of this paper was to design a full adder with high speed performance using GDI technique. From the performance analysis table it is clear that the proposed design system is the best among the discussed designs in terms of area, delay and power dissipation. Since the results 
were obtained as an outcome of simulation, the readings are precise. This design will have an improved speed and also the efficiency of the system is more compared to all the conventional techniques. Further modifications can be made in the design by adding a few more transistors.

\section{References}

[1] K. Navi, M.H. Moaiyeri, R. FaghihMirzaee, O. Hashemipour, B. MazloomNezhad, Two new low-power full adders based on majority-not gates, Microelectronics Journal (Elsevier), 40, 126130.

[2] M.H. Moaiyeri, R. FaghihMirzaee, K. Navi, T. Nikoubin, O. Kavehei, Novel direct designs for 3input XOR function for low power and high-speed applications, International Journal of Electronics (Taylor and Francis) 97 (6) (2010) 647-662.

[3] K. Navi, M. Maeen, V. Foroutan, S. Timarchi, O. Kavehei, A novel low power full-adder cell for low voltage, Integration the VLSI Journal (Elsevier) 42 (4) (2009) 457-467.

[4] M. Alioto, G. Palumbo, Analysis and comparison of the full adder block, IEEE Transactions on VLSI 10 (6) (2002) 806-823.

[5] C.H. Chang, J. Gu, M. Zhang, A review of 0.18 um full-adder performances for tree structure arithmetic circuits, IEEE Transactions on Very Large Scale Integration(VLSI)Systems 13 (6) (2005).

[6] A.M. Shams, T.K. Darwish, M.A. Bayoumi, Performance analysis of low-power 1-bit CMOS full adder cells, IEEE Transactions on Very Large Scale Integration (VLSI) Systems 10 (1) (2002) 20-29.

[7] Arkadiy Morgenshtein, A. Fish, Israel A. Wagner, Gate-diffusion input (GDI): a power-efficient method for digital combinatorial circuits, IEEE Transactions on VLSI Systems (2002) 566-581.

[8] R. Zimmermann, W. Fichtner, Low-power logic styles: CMOS versus pass- transistor logic, IEEE Journal of Solid-State Circuits 32 (1997) 1079-1090.

\footnotetext{
*Corresponding author.

E-mail address: mesairsolomon@ gmail.com
} 